\title{
Perfil de súper-corrientes en una lámina de $A l$ a campo magnético cero
}

\section{Super-current profile in an $A l$ flat at zero magnetic field}

\section{Perfil de super-correntes em uma lâmina de $A l$ a campo magnético zero}

\author{
Fernando Duran-Flórez ${ }^{1}$, Miryam Rincón-Joya², José Barba-Ortega ${ }^{3}$
}

Forma de citar: F. Duran-Flórez, M. Rincón-Joya, J. Barba-Ortega, "Perfil de súper-corrientes en una lámina de $A l$ a campo magnético cero", Respuestas, vol. 21, no. 2, pp. 6-12, 2016.

Recibido:

Enero 12 de 2016

Aceptado:

Abril 22 de 2016

${ }^{1}$ Magister en Fisica fernando.duran@upb.edu.co Orcid: 0000-0002-2457-6691

Universidad Pontificia Bolivariana

Bucaramanga-Colombia

${ }^{2}$ Doctorado en física mrinconj@unal.edu.co Orcid: 0000-0002-4209-16

Universidad Nacional de Colombia

Departamento de Física Bogotá-Colombia

${ }^{3}$ Doctorado en Física jjbarbao@unal.edu.co Orcid: 0000-0003-3415-1811

Universidad Nacional de Colombia

Departamento de Física Bogotá-Colombia

\section{Resumen}

Objetivo: En este artículo se presentan resultados numéricos de simulaciones computacionales del perfil de supercorrientes en una placa superconductora de aluminio a baja temperatura, visando a ser analizados para la fabricación de nanoestructuras para posibles aplicaciones en dispositivos nano-electrónicos. Metodología: Se solucionan las ecuaciones dependientes del tiempo Ginzburg-Landau con el popular método de variables de enlace para una película de Aluminio con una corriente directa aplica y en ausencia de campo magnético aplicado. Resultados: Pares de vórtices cinemáticos emergen de la parte central de la muestra con su posterior aniquilación en un valor determinado de la corriente. La nucleación periódica de los vórtices cinemáticos conduce a la aparición de oscilaciones temporales del voltaje. Hacia corrientes altas el estado superconductor es destruido y la muestra se comporta como un metal óhmico. Hacia corrientes bajas una pequeña resistencia aparece debida a los contactos metálicos donde la corriente es aplicada. Conclusión: La aparición de pares vórtices-anti-vórtices destruye el estado superconductor en la parte media de la lámina. Su naturaleza es determinada por el perfil de supercorriente dentro de la muestra. Vórtices cinemáticos muestran una geometría alongada ocasionando un aumento drástico en la resistencia local del material.

Palabras clave: Ginzburg-Landau, Mesoscópico, Superconductor, Vórtices Cinemáticos

\begin{abstract}
Objective: This paper presents numerical results of computational simulations of the Supercurrent profile in a superconducting aluminum flat at low temperature, aiming to be analyzed for the fabrication of nanostructures for possible applications in nanoelectronics devices. Methodology: Time dependent Ginzburg-Landau equations were solved with the popular Link variable method for aluminum flat with a direct applied current and in absence of external applied magnetic field. Results: Kinematics vortex pairs emerge of the middle part of the sample and a subsequent annihilation in a determined value for the applied current. The periodic vortex kinematic pair nucleation leads to the appearance of temporal voltage oscillations. At higher current, the superconducting state is completely destroyed and the sample behaves as a ohmic metal. At lower current a low resistance is present due to metallic contact where the current is applied. Conclusion: The appearance
\end{abstract}


Fernando Duran-Flórez, Miryam Rincón-Joya, José Barba-Ortega

of vortex-anti-vortex pairs destroys the superconducting state in the middle of the sheet. Its nature is determined by the supercurrent profile within the sample. Kinematic vortex shows an elongated geometry causing a drastic increase in local resistance of the material.

Keywords: Ginzburg-Landau, Mesoscopic, Superconductor, Kinematics vortex.

\title{
Resumo
}

\begin{abstract}
Objetivo: Neste artigo se apresentam resultados numéricos de simulações computacionais do perfil de super-correntes numa placa supercondutora de alumínio a baixa temperatura, visando a ser analisados para a fabricação de nanoestruturas para possíveis aplicações em dispositivos nano-electrónicos. Metodologia: Solucionaram-se as equações dependentes do tempo Ginzburg-Landau com o popular método de variáveis de enlace para uma película de Alumínio com uma corrente direta e em ausência de campo magnético aplicado. Resultados: Pares de vórtices cinemáticos emergiram da parte central da amostra com sua posterior aniquilação num valor determinado da corrente. A nucleação periódica dos vórtices cinemáticos conduziu à aparição de oscilações temporais da voltagem. A altas correntes o estado supercondutor foi destruído e a amostra se comportou como um metal ôhmico. A baixas correntes uma pequena resistência apareceu, devida aos contatos metálicos onde a corrente foi aplicada. Conclusão: A aparição de pares vórticesanti-vórtices destruiu o estado supercondutor na parte do meio da lâmina. Sua natureza foi determinada pelo perfil de super-corrente dentro da amostra. Vórtices cinemáticos mostraram uma geometria alongada ocasionando um aumento drástico na resistência local do material.
\end{abstract}

Palavras-chave: Ginzburg-Landau, Mesoscópico, Supercondutor, Vórtices Cinemáticos.

\section{Introducción}

La dinámica de fluxoides magnéticos en superconductores de baja temperatura crítica tiene muchas aplicaciones tecnológicas e industriales ya que este tipo de materiales presenta resistividad eléctrica nula bajo ciertos parámetros críticos como temperatura, campo magnético y corriente. La manipulación de este tipo de flujos magnéticos cuantizados es de vital importancia ya que, la existencia de vórtices en el material origina calor Joule, destruyendo localmente la superconductividad con la respectiva consecuencia de disipación de calor en los elementos electrónicos. Unos de los objetivos de la ingeniería actual es limitar y hasta eliminar la pérdida de energía en elementos electrónicos y llevar a la fabricación de nuevos dispositivos como elementos lógicos, SQUIDs, detectores de fotones, dispositivos de resonancia magnética nuclear, tomografía óptica, generación de altos campos magnéticos, levitación magnética, etc.
[1]-[10]. De una forma diferente a los llamados vórtices Abrikosov, los cuales son flujos de campo magnético cuantizado, existe un tipo de vórtice muy interesante llamado vórtice cinemático, este vórtice se origina debido a corrientes externas aplicadas generando campos magnéticos (Ley de Ampere) dentro de la muestra que, debido a su dirección dentro de la misma originan un par vórtice-antivórtice $(\mathrm{V}-\mathrm{Av})$. La velocidad de creación y aniquilación del par V-Av es extremadamente rápida por lo cual es muy difícil apreciarlos como fluxoides independientes. Físicamente estos vórtices cinemáticos son puntos en el espacio-tiempo donde la pseudo-funcion de onda superconductora es igual a cero, su fase muda un valor igual en torno de ese punto, $\mathrm{N}$ indica la vorticidad o número de cuantos de flujo magnético. Una gran cantidad de trabajos experimentales y teóricos se han desarrollado desde la descubierta del fenómeno de la superconductividad, una de las primeras aplicaciones tecnológicas fue en el logro en
Cúcuta-Colombia

Vol. 21

No. 2

Jul - Dic 2016

ISSN 0122-820X

E-ISSN 2422-5053

PP: 6-12

(2)

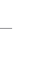


la fabricación de nanoestructuras magnéticassuperconductoras-aislantes como centros de anclajes en películas delgadas, esto conllevó a que estos materiales permanecieran en el estado superconductor a mayores corrientes aplicadas [11], [12]. En presencia de corrientes externas, se han realizados muchas investigaciones entre ellos podemos citar a M. V. Milosevic, quien estudió el efecto que la fuerza de Lorentz en superconductores con defectos magnéticos, encontró que la corriente crítica aumenta considerablemente con la inclusión de estos magnetos [13]. G. Berdiyorov, estudió la respuesta magnética de una lámina de Niobio en ausencia de campo magnético variando la temperatura y en presencia de corriente aplicada, encontró que el flujo de los fluxoides es estable a bajas temperaturas. En el estado de vórticesanti-vórtices varios autores han mostrado teóricamente que los vórtices Abrikosov dentro de una cavidad Josephson generan pares $\mathrm{V}-\mathrm{Av}$ y en una lámina delgada y en ausencia de campo magnético externo aparecen los llamados vórtices cinemáticos [14], [15], [16]. P. Sanchez, analizó la resistividad que presenta el estado de movimiento de los vórtices Abrikosov en láminas superconductoras variando su tamaño, encontró un anclaje de estos vórtices en la superficie de la muestra con la consecuente aparición de voltaje. Un resultado muy importante y reciente fue reportado por T. Golod, el autor probo experimentalmente que un vórtice Abrikosov puede ser analizado como una célula de memoria de acceso aleatorio y usado como un bit informativo, estas células se caracterizan por tener una energía baja de grabación y magneto-resistencia gigante entre los estados uno y cero en un corto tempo de acceso [17]. En este trabajo solucionamos el sistema de ecuaciones acopladas dependientes del tempo Ginzburg-Landau en una placa superconductora de Aluminio puro a baja temperatura en presencia de una corriente directa y en ausencia de campo magnético externo. Para un valor determinado de la corriente encontramos creación y aniquilación de pares vórtices-anti-vórtices. Mostramos que estos pares de vórtice aparecen o nuclean a los lados perpendiculares a la aplicación de la corriente, siguiendo la convención de Ampere, en la mitad de la placa en su centro. Si se tiene un sistema en presencia de campo magnético esta dinámica de pares $\mathrm{V}$-Av difiere drásticamente, aparecerán a su vez vórtices Abrikosov y los puntos de creación de los vórtices dinámicos estarán determinados por la distribución de supercorrientes en el sistema [16].

\section{Materiales y métodos}

\subsection{Formalismo Teórico}

Unadelasteoríasconmáséxitoenladescripción de superconductores de baja temperaturas crítica es la teoría fenomenológica de Ginzburg-Landau (TGL). Esta teoría describe el estado superconductor por medio de una pseudo función de onda compleja $\psi(r, t)$ cuyo modulo cuadrado representa la densidade de eléctrones superconductores y del potencial vector $\boldsymbol{A}(r, t)$. Las ecuaciones GinzburgLandau en presencia de corrientes aplicadas relacionadas con el potencial electrostático $\varphi$ a temperatura $T=0$ han sido ampliamente estudiadas [8],[10],[16],[18]. Los puntos donde la corriente externa J es aplicada son contactos metálicos, donde $\Psi=0$ e $\nabla \varphi=-\mathrm{J}$. Las condiciones de contorno de Neumann fueron utilizadas en la región donde la corriente es aplicada $\mathrm{J}_{\perp}=\left.\hat{n} \cdot(-i \nabla+\boldsymbol{A}) \Psi\right|_{n}=0$. La muestra estudiada es una película superconductora delgada de $A l\left(\left(\xi_{0}\right)=100 \mathrm{~nm}\right)$ [19] de área transversal $a \times b$, localizada no plano $(x, y)$ a campo magnético cero.

\section{Resultados y análisis}

Los parámetros computacionales usados que garantizan una buena convergencia numérica son, tamaño de la muestra $a \times b=12 \xi \times 8 \xi$, siendo el tamaño real $1200 \mathrm{~nm} \times 800 \mathrm{~nm}$ (Figura 1), tamaño de la malla computacional, $\Delta=\Delta=0.1$, paso de la corriente aplicada $\Delta J=10^{-3}$, paso temporal $\Delta t=0.001$, tamaño del contacto donde se aplica la corriente $W=2 \xi=$ $200 \mathrm{~nm}$, parametro Ginzburg-Landau $K=5.0$. 


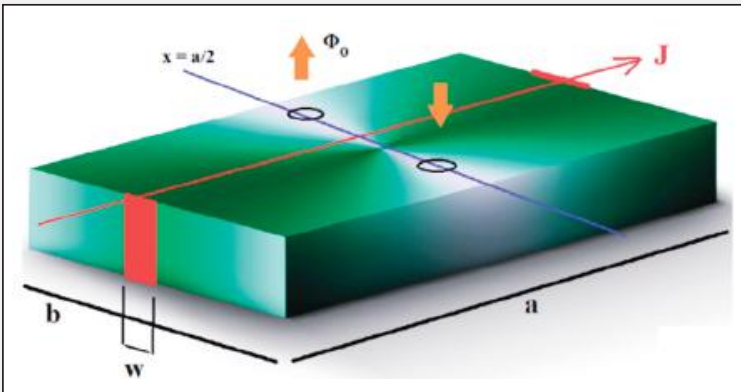

Figura 1. Esquema de la muestra estudiada, $\boldsymbol{J}$ es la densidade de corriente aplicada, $\Phi_{0}$ es el quanto de flujo magnético, $\boldsymbol{W}$ es la dimensjon de los contatos metálicos, $\boldsymbol{a} \boldsymbol{x} \boldsymbol{b}$ es la dimension de la muestra.

Fuente: Autores

En las Figuras 2 y 3 se gráfica el perfil de la corriente aplicada y de la supercorriente que rodea al núcleo del vórtice (abajo) $\mathrm{y}$ al anti vórtice (arriba), la dirección de las supercorrientes es horaria y antihoraria respectivamente a través de la línea cinemática.
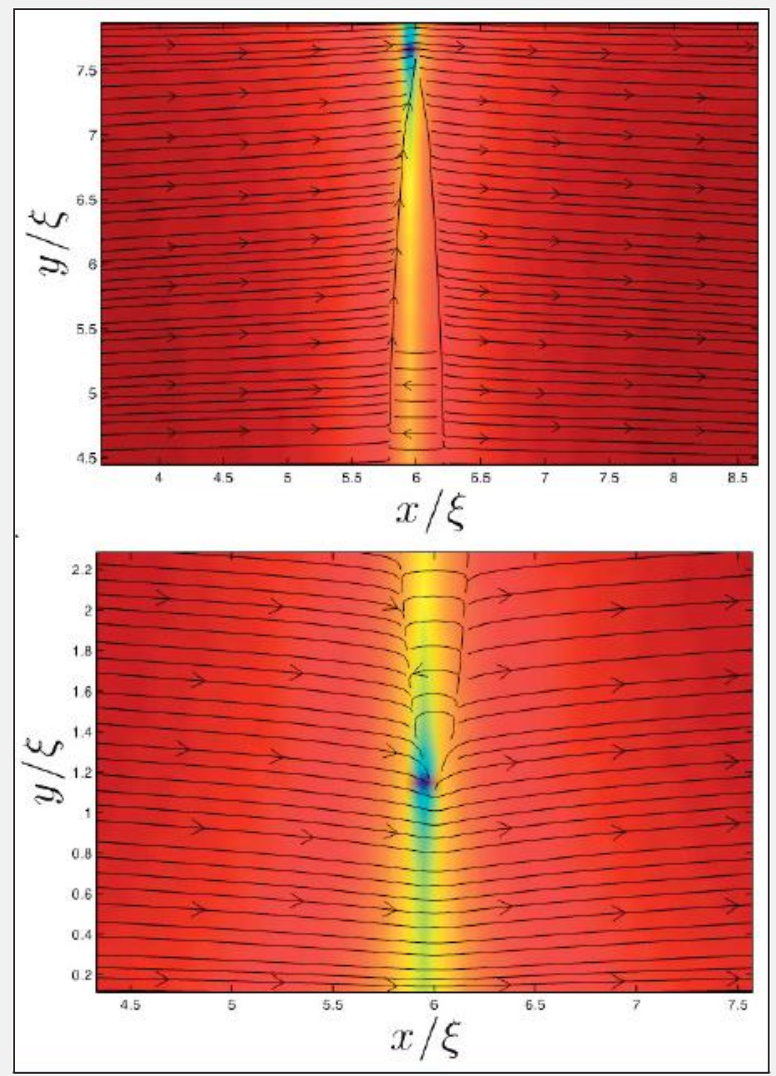

Figura 2. Logaritmo del parámetro de orden superconductor y perfil de la corriente aplicada y de la supercorrientes en el centro de la lámina, para una densidad de corriente aplicada $J=1.50$ en un tiempo $t=2421$

Autores

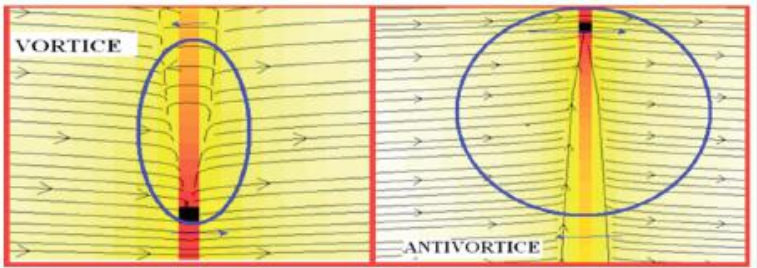

Figura 3. Iguales valores que en la Figura 2, incluyendo la circulación esquemática de la corriente (línea azul) para un vórtice con circulación anti-horaria (izquierda) y un anti vórtice con circulación horaria (derecha) Fuente: Autores

La distribución de corriente a través de la muestra está más influenciada por la configuración de V-Av que por la distribución uniforme de la corriente externa aplicada, a su vez la velocidad del par V-Av puede verse influenciada por la presencia de campos magnéticos externos, favoreciendo el flujo de los vórtices y oponiéndose a los anti-vórtices. La velocidad de los vórtices es inversamente proporcional a la magnitud del campo magnético aplicado [16].

En la Figura 4 se gráfica el perfil de la corriente aplicada dentro de la muestra y de la supercorriente que rodea al núcleo del par V-Av para una densidad de corriente aplicada de $J=1.50$ durante un intervalo de tiempo desde $t_{1}=2421$ hasta $t_{2}=2457$, se puede apreciar que el sistema transita en diferentes estados de $\mathrm{V}-\mathrm{Av}$, los cuales son creados en el centro de la lámina y se desplazan hacia los bordes de la muestra bajo la influencia de la corriente aplicada, después de la expulsión de este par, un nuevo par es creado otra vez en la mitad central de la lámina, continuando el proceso cinemático. Esta nucleación periódica permite la existencia de oscilaciones del voltaje en la muestra (Figura 5). Como es de esperarse con un incremento en la corriente la velocidad de este proceso incrementa pero no alcanza nunca la misma velocidad de creación V-Av. 
Vol. 21

No. 2

Jul - Dic 2016

ISSN 0122-820X

E-ISSN 2422-5053

PP: 6-12

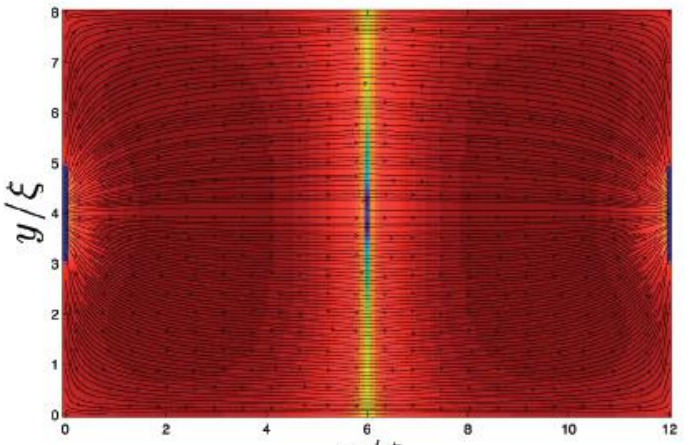

$x / \xi$

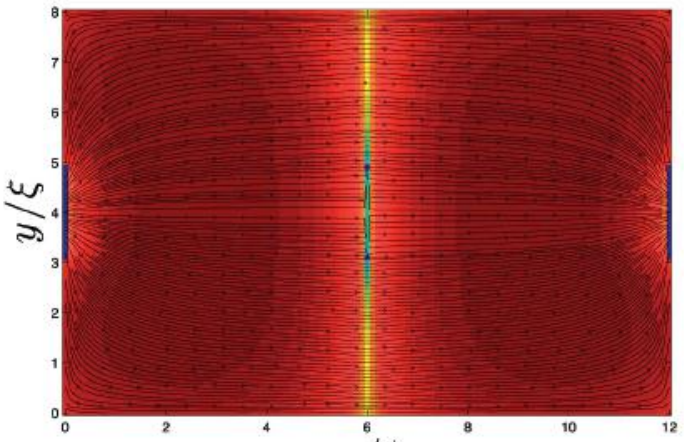

$x / \xi$
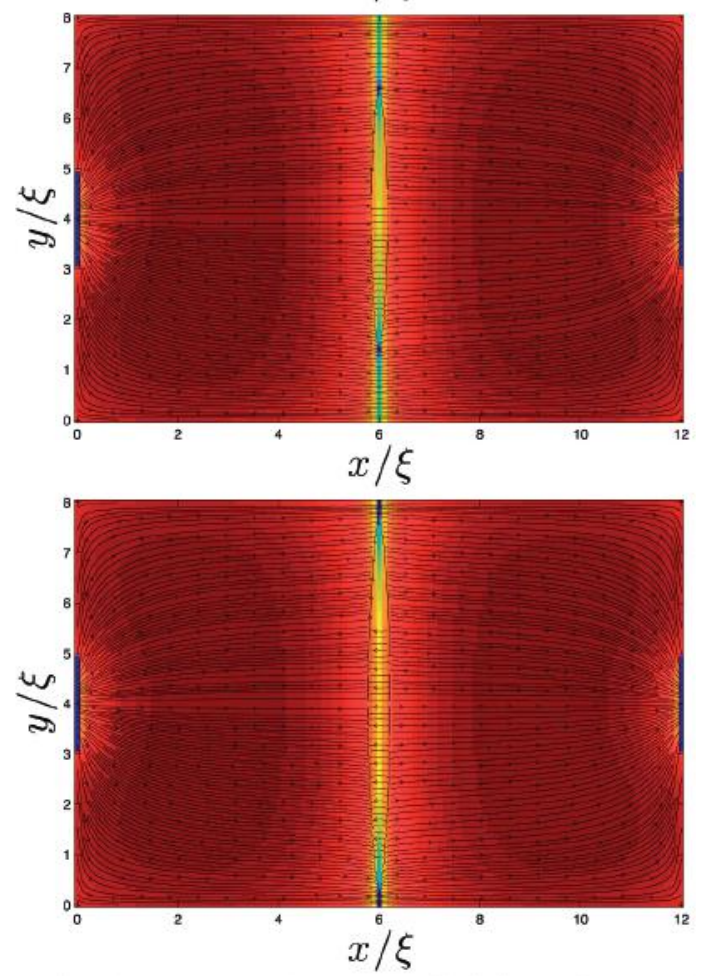

Figura 4. Logaritmo del parámetro de orden superconductor y perfil de la corriente aplicada $J=1.50$ y de la supercorriente en el centro de la lámina, para una densidad de corriente aplicada y un tiempo $t=2421,2428,2445,2457$.

Fuente: Autores

En la Figura 5 graficamos la Curva de voltaje en función del tempo para una densidad de corriente aplicada $J=1.50$ en varias ampliaciones de arriba hacia abajo, observando el eje vertical podemos apreciar el recuadro ampliado. Podemos concluir que máximos y mínimos del voltaje corresponde a la nucleación y aniquilación de pares V-Av, la situación es bastante interesante, ya que el tiempo de vida de los pares $\mathrm{V}$-Av es mucho menor que el período de las oscilaciones del voltaje.
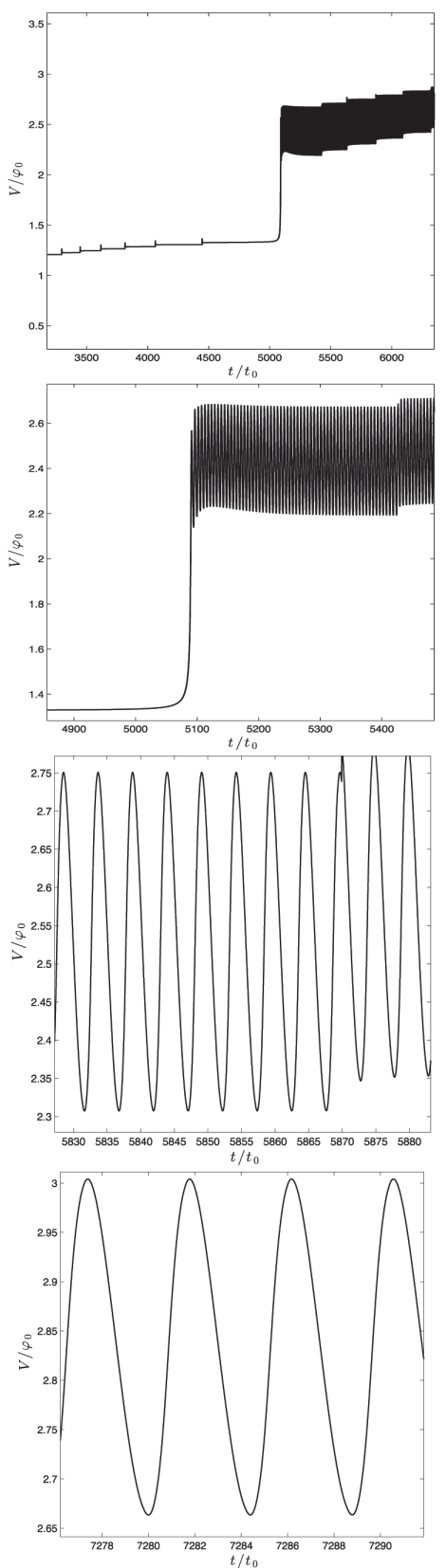

Figura 5. Curva de voltaje en función del tempo para una densidad de corriente aplicada $J=1.50$. De Arriba hacia abajo ampliación, observando los ejes vertical/horizontal se aprecia el intervalo estudiado Fuente: Autores 


\section{Conclusiones}

Resolviendo las ecuaciones dependientes del tempo Ginzburg-Landau, estudiamos la aparición y aniquilación de pares vórticesanti-vórtices en una lámina de aluminio en ausencia de campos magnéticos. Encontramos que el estado resistivo es representado por una pequeña franja en el medio de la muestra donde los pares vórticeanti-vórtice nuclean. Los pares V-Av nuclean en el centro de la muestra y se mueven hacia las fronteras exteriores donde se aniquilan en una resonancia periódica. La nucleación periódica de los vórtices cinemáticos permite la existencia de oscilaciones perfectas del voltaje en la muestra. La posición de los puntos de aniquilación - creación podría ser manipulada incluyendo campos magnéticos externos en los puntos donde los vórtices cinemáticos emergen en la muestra obteniendo dos especies de vórtices, cinemáticos y de Abrikosov, siendo una gran posibilidad de futuras aplicaciones.

\section{Agradecimientos}

Los autores agradecen a Edson Sardella por sus útiles discusiones.

\section{Referencias}

[1] J. Van de Vondel, C. C. de Souza, B. Y. Zhu, et al. "Vortex-Rectification Effects in Films with Periodic Asymmetric Pinning", Phys. Rev. Lett., vol. 94, no. 5, pp. 057003, 2005.

[2] K. Yu, T. W. Heitmann, C. Song, et al. "Asymmetric weak-pinning superconducting channels: Vortex ratchets", Phys. Rev. B, vol. 76, no. 22, pp. 220507, 2007.

[3] K. Yu, M. B. S. Hesselberth, P. H. Kes and B. L. T. Plourde. "Vortex dynamics in superconducting channels with periodic constrictions", Phys. Rev. B, vol 81, pp. 184503, 2010.
[4] M. B. Hastings, C. J. O. Reichhardt and C. Reichhardt. "Ratchet Cellular Automata”, Phys. Rev. Lett., vol 90, no. 24, pp. 247004, 2003.

[5] T. Puig, E. Rosseel, M. Baert, et al. "Stable vortex configurations in superconducting $2 \times 2$ antidot clusters", Appl. Phys. Lett., vol 70, pp. 3155, 1997.

[6] G. Karapetrov, V. Yefremenko, G. Mihajlovic, et al. "Evidence of vortex jamming in Abrikosov vortex flux flow regime", Phys. Rev. B, vol. 86, no. 5, pp. 054524, 2012.

[7] G. Berdiyorov, K. Harrabi, F. Oktasendra, et al. "Dynamics of current-driven phase-slip centers in superconducting strips", Phys.Rev. B, vol. 90, no. 5, pp. 054506, 2014.

[8] F. Rogeri, R. Zadorosny, P. N. LisboaFilho, et al. "Magnetic field profile of a mesoscopic SQUID-shaped superconducting film", Supercond. Sci. Technol., vol. 26, no. 7, pp. 075005, 2013.

[9] R. I. Rey, A. R. Alvarez, C. Carballeira, et al. "Measurements of the superconducting fluctuations in optimally doped $\mathrm{BaFe}_{2}-\mathrm{xNixAs}_{2}$ under high magnetic fields: probing the 3D-anisotropic Ginzburg-Landau approach", Supercond. Sci. Technol., vol. 27, no. 7, pp. 07500, 2014.

[10] P. J. Pereira, V. V. Moshchalkov and L. F. Chibotaru. "Efficient solution of 3D Ginzburg-Landau problem for mesoscopic superconductors". J. Phys. Conf. Ser, vol. 490, pp. 012220, 2014.

[11] J. I. Martin. "Flux Pinning in a Superconductor by an Array of Submicrometer Magnetic Dots", Phys. Rev. Lett., vol. 79, no. 10, pp. 1929, 1997.
Jul - Dic 2016

ISSN 0122-820X

E-ISSN 2422-5053

PP: 6-12 
No. 2

Jul - Dic 2016 ISSN 0122-820X E-ISSN 2422-5053 PP: 6-12

[12] D. J. Morgan and J. B. Ketterson. "Asymmetric Flux Pinning in a Regular Array of Magnetic Dipoles", Phys. Rev. Lett., vol. 80, no.16, pp. 3614, 1998.

[13] M. V. Milosevic, G. R. Berdiyorov and F. M. Peeters, Phys. "Mesoscopic Field and Current Compensator Based on a Hybrid Superconductor-Ferromagnet Structure", Rev. Lett., vol. 95, no. 14, pp. 147004, 2005.

[14] G. R. Berdiyorov. "Effect of surface functionalization on the electronic transport properties of $\mathrm{Ti}_{3} \mathrm{C}_{2}$ MXene", Eurp. Phys. Lett., vol. 111, no. 6, pp. 67002, 2015.

[15] G. R. Berdiyorov, K. Harrabi, J. P. Maneval and F. M. Peeters. "Effect of pinning on the response of superconducting strips to an external pulsed current", Supercond. Sci. Technol, vol. 28, no. 2, pp. 25004, 2015.

[16] G. R. Berdiyorov, M. V. Milosevic, F. M. Peeters and D. Y. Vodolazov. "Kinematic vortex-antivortex lines in strongly driven superconducting stripes", Phys. Rev. B, 79, no. 18, pp. 184506, 2009.

[17] P. Sanchez, J. Albino Aguiar and D. Dominguez. "Behavior of the flux-flow resistivity in mesoscopic superconductors", Physica C, vol. 503, pp. 120-122, 2014.

[18] D. Gropp, H. G. Kaper, G. K. Leaf, et al. "Numerical simulation of vortex dynamics in type-II superconductors", J. Comput. Phys., Vol. 123, no. 2, pp. 254-266, 1996.

[19] C. P. Poole Jr. "Superconductivity", 2nd, Elsevier Academic Press, The Netherlands, 2007. 